\title{
Adding Seebeck coefficient measurements to an existing high temperature device for Hall constant and electrical conductivity measurements
}

\author{
Robin Werner ${ }^{1}$, Jaroslaw Kita ${ }^{1}$, Michael Gollner ${ }^{2}$, Florian Linseis ${ }^{2}$, Ralf Moos ${ }^{1}$ \\ ${ }^{1}$ Department of Functional Materials, University Bayreuth, 95440 Bayreuth, Germany \\ 2 Linseis Thermal Analysis, 95100 Selb, Germany \\ functional.materials@uni-bayreuth.de
}

\begin{abstract}
Summary:
An already existing high temperature device to determine the electrical conductivity and the Hall constant has been extended for thermoelectric Seebeck coefficient measurements. The sample holder made in thick-film technology is equipped with two platinum heaters. Whereas the primary heater allows to reach the desired measuring temperature, the second one allows measurements at variable temperature differences. Measurements on constantan, a certified reference material, confirm the functionality of the Seebeck measurements with this new measurement setup.
\end{abstract}

Keywords: Seebeck coefficient, Hall constant, electrical conductivity, high temperature, low-cost

\section{Motivation}

For electrical material characterization, the electrical conductivity, the carrier density, the carrier mobility, and the Seebeck coefficient are of great importance. Especially challenging is the characterization at high temperatures. Current commercial instruments can either determine the electrical conductivity and the Hall constant or determine the electrical conductivity and the Seebeck coefficient. Often, expensive and slow furnaces are needed. Furthermore, expensive electromagnets are used. A novel sample holder design developed with the help of FEM simulations and manufactured in thick-film technology, makes it possible to characterize all of the above-mentioned parameters within one measuring device and eliminates the need for expensive furnaces and electromagnets. This contribution describes an extension of an existing system for thermoelectric Seebeck coefficient measurements.

\section{Description of the New System}

The basic structure of the new sample holder is a $635 \mu \mathrm{m}$ thick alumina ceramic substrate with four electrodes on the upper side. The electrodes are spring mounted and moveable, which allows to contact samples of any geometry within a sample area with a maximum diameter of $12.7 \mathrm{~mm}$ according to the van der Pauw measuring method [1]. Gold screen-printed conductor lines and the contact pads are located on the reverse side of the sample holder. A screw-nut combination with an integrated spring serves as a through-hole connection between both sides of the substrate. For connection with the electronics, a standard card-edge connector is used.

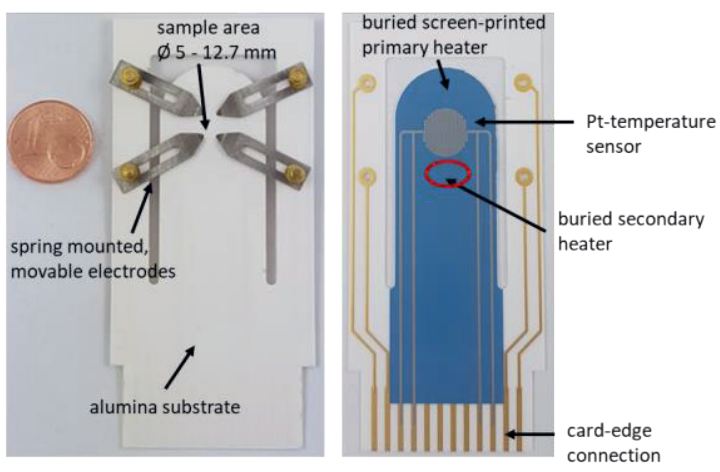

Fig. 1. Sample holder for conductivity, Hall constant and thermoelectric Seebeck measurements with two buried heaters

Fig. 1 shows the front and reverse side of the sample holder. Besides the mentioned gold conductors and contacts, a screen-printed platinum resistive temperature sensor is visible. For precise resistance measurement, a four-wire technique is used. The temperature in the sample area is determined via a four-wire resistance measurement by a previously done one-time resistance-temperature calibration. The temperature within this area is generated by two platinum heaters located under the blue cover layer. The desired measuring temperature can be generated by Joule heating via the primary heater. The secondary heater can be used to generate a variable temperature difference within the sample area. The necessary measurement of the con- 
tact point temperature, as well as the thermoelectric voltage for Seebeck measurements is realized by gold-platinum thermocouples, which can be clamped between the electrode tip and the sample.

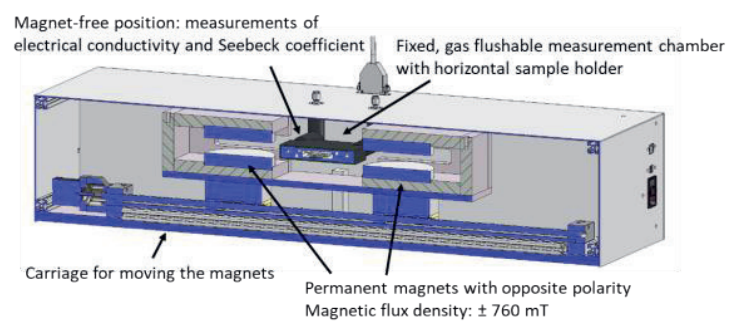

Fig. 2. Measurement setup: two movable permanent magnetic yoke systems with +/- $760 \mathrm{mT}$ and a magnet-free position for electrical conductivity and thermoelectric Seebeck measurements

The presented sample holder, including all components, has a height of only a few millimeters and can be installed inside a small gas flushable aluminum measuring chamber that can be seen in Fig. 2. The system additionally contains two magnetic yoke systems made of permanent magnets with different polarity, with which the electrical conductivity and the charge carrier density could be measured up to $600{ }^{\circ} \mathrm{C}$ as described in [2].

\section{Seebeck Coefficient Measurements}

For the measurements of the Seebeck coefficient and to test the functionality of the new system, a sample of constantan was chosen, which is well known in the thermoelectric community as a reference material. For the measurements, the sample holder was heated up to $700{ }^{\circ} \mathrm{C}$ in $100{ }^{\circ} \mathrm{C}$ steps by the primary heater. Afterwards, the temperature difference over the sample was varied by varying the secondary heater. The resulting thermoelectric voltage within the sample is measured via the platinum wires of the goldplatinum thermocouples. The Seebeck coefficient can be calculated from the ratio of the thermoelectric voltage and the temperature difference between the thermocouples, considering the temperature of the reference junction and the thermoelectric voltage of the platinum wire. Fig. 3 shows experimental results of the absolute Seebeck coefficient measurements up to $700{ }^{\circ} \mathrm{C}$. The Seebeck coefficient increases with increasing temperature. The measurement was performed twice, with an intermittent sample change. The measurements show the same trend as the known values of the certificate. Also, the absolute values differ by only a few percent, which is why the functionality of the measurement setup can be called functional.

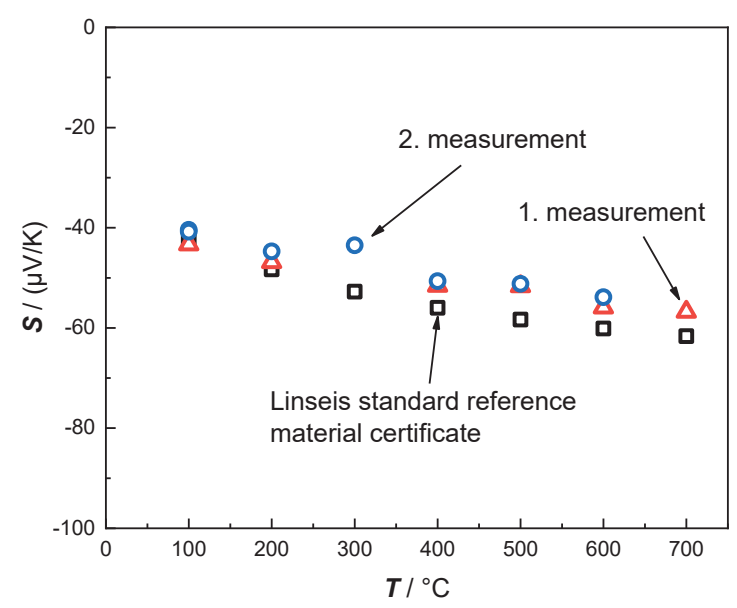

Fig. 3. Measurement of the Seebeck coefficient of constantan up to $700{ }^{\circ} \mathrm{C}$

\section{Conclusion and Outlook}

By adding a second screen-printed heater and two gold-platinum thermocouples, an already existing measurement setup for the measurement of the Hall constant and the electrical conductivity could be extended by an arrangement to determine the Seebeck coefficient. Measurements on constantan as a certified reference material with known temperature dependent Seebeck coefficient have shown that the Seebeck coefficient can be determined up to $700{ }^{\circ} \mathrm{C}$ with the hereinvestigated setup.

In future, all mentioned material parameters will be determined in one measurement cycle to validate the functionality of this new combined lowcost measurement instrument. Furthermore, a continuous increase of the maximum measuring temperature is envisaged.

\section{References}

[1] L.J. van der Pauw, A Method of Measuring Specific Resistivity and Hall Effect of Discs of Arbitrary Shape, Semiconductor Devices: Pioneering Papers, 174-182 (1991); doi: 10.1142/9789814503464_0017

[2] R. Werner, J. Kita, M. Gollner, F. Linseis, R. Moos, Development of a new Measurement System for Electrical Conductivity and Hall Constant, SMSI Sensor and Measurement Science International, cancelled conference, 22-25 June 2020, Nuremberg, Germany, doi: 10.5162/SMSI2020/A5.4

\section{Acknowledgements}

The presented results have been achieved in the scope of the project ESB-1607$0002 / / E S B 040 / 001$. It has been funded by the Bavarian Ministry of Economic Affairs and Media, Energy and Technology, VDI/VDE-IT Electronic Systems Program. 